\title{
IARS2 regulates proliferation, migration, and angiogenesis of human umbilical vein endothelial cells
}

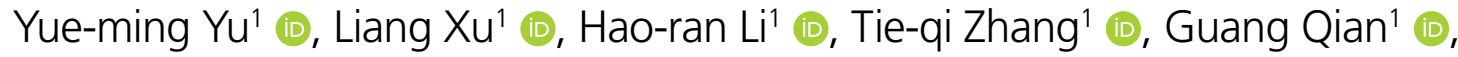 \\ Ling-feng Li ${ }^{1} \mathbb{B}$, Ming-hai Wang ${ }^{1 *}$ (C)
}

\begin{abstract}
SUMMARY
OBJECTIVE: In this study, we aimed at investigating the role of isoleucyl-tRNA synthetase in the growth, migration, and angiogenesis of human umbilical vein endothelial cells and the underlying molecular mechanism.

METHODS: To assess the role of isoleucyl-tRNA synthetase, we silenced isoleucyl-tRNA synthetase in human umbilical vein endothelial cells using lentiviral 2 specific short hairpin RNAs (short hairpin RNAs 1 and 2) and examined silencing efficiency using real time quantitative polymerase chain reaction and western blot analyses. Short hairpin RNAs 1- isoleucyl-tRNA synthetase had greater knockdown efficiency, it was used in the entire downstream analysis. Short hairpin RNAs 1-isoleucyl-tRNA synthetase silencing effects on cell proliferation, cell colony generation, cell migration, as well as angiogenesis were assessed using cell counting kit-8, colony development, cell migration, and angiogenesis tube formation assays, respectively.

RESULTS: Compared to the control group, anti- isoleucyl-tRNA synthetase short hairpin RNAs significantly silenced isoleucyl-tRNA synthetase expression in human umbilical vein endothelial cells, and suppressed their proliferation, migration, and angiogenic capacity. To characterize the underlying mechanism, western blot analyses showed that isoleucyl-tRNA synthetase knockdown suppressed phosphorylation of extracellular-regulated kinase $1 / 2$ and protein-serine- threonine kinase, as well as expression of vascular endothelial growth factor, GSK-3 $\beta$, and $\beta$-catenin.

CONCLUSIONS: We have shown, for the first time, the critical role of isoleucyl-tRNA synthetase in human umbilical vein endothelial cells. Our data show that isoleucyl-tRNA synthetase knockdown suppresses human umbilical vein endothelial cell proliferation, migration, and angiogenesis. We have also shown that isoleucyl-tRNA synthetase knockdown suppresses phosphorylation of extracellular-regulated kinase $1 / 2$ and protein-serine- threonine kinase, as well as expression of vascular endothelial growth factor, GSK-3 $\beta$, and $\beta$-catenin. Together, these data highlight isoleucyl-tRNA synthetase as a potential antitumor anti-angiogenic target.

KEYWORDS: Human umbilical vein endothelial cells. Isoleucine-tRNA ligase. Angiogenic proteins. Cell proliferation. Cell migration
\end{abstract}

\section{INTRODUCTION}

Angiogenesis, the physiological process through which new blood vessels are formed, provides oxygen and nutrients to actively proliferating tumor cells ${ }^{1}$. To support the high proliferative degree of cancer cells, tumors should rapidly form new vascular networks ${ }^{2}$. Multiple clinical studies show that tumors are angiogenesis dependent. This process is needed to support the growth of tumors beyond $1-2 \mathrm{~mm}^{3}$. Thus, anti-angiogenesis has arisen as a prospective anti-cancer approach ${ }^{3}$. Angiogenesis is strongly modulated by an equilibrium between pro- and anti-angiogenic factors. Multiple factors like VEGF, neuropilin-1, tyrosine kinase with Ig, and bFGF are involved in angiogenesis. Elevated tumor VEGF levels are a key feature in tumor angiogenesis ${ }^{4}$. While multiple promising anti-angiogenic therapy

\footnotetext{
'Fudan University, The Fifth People's Hospital of Shanghai, Department of Orthopedics - Shanghai, China.

*Corresponding author: king1972@163.com

Conflicts of interest: The authors declare there is no conflicts of interest. Funding: This study was supported by the Fifth People's

Hospital of Shanghai (No. 2020WYFY01, 2018WYZT01).

Received on November 26, 2020. Accepted on December 13, 2020.
} 
trials have been carried out, outcomes have been unsatisfactory 5 . Thus, profound comprehension of the molecular basis of angiogenesis is needed to design potential anti-angiogenic therapies.

Aminoacyl-tRNA synthetases (ARSs) constitute a family of 20 essential enzymes (one for each amino acid) that bind amino acids to their respective tRNAs during protein biosynthesis ${ }^{6}$. In higher eukaryotes ARSs have been shown to be critical for cell growth, differentiation, cytokine activity, tumorigenesis, and angiogenesis ${ }^{7}$.

IARS2 (isoleucyl-tRNA synthetase), which belongs to class I mitochondrial ARS family, is a 1012 amino acid protein found in the mitochondrial matrix. IARS2 is documented to enhance cell proliferation, inhibit apoptosis, and enhance tumor ability to form RKO cells ${ }^{8}$. IARS2 knockdown suppresses cell proliferation, cell colony formation, and triggers cell cycle interruption in the gastric cancer cell line, AGS 9 . Taken together, these reports implicate IARS2 in tumor progression. However, the role of IARS2 in angiogenesis remains uncertain. In this study, we used shRNA to silence IARS2 in HUVECs and investigated its effects and underlying mechanisms in modulating proliferation, migration, and angiogenesis.

\section{METHODS}

\section{Cell culture}

We obtained HUVEC and HEK293T cells from the cell bank of the Chinese Academy of Science (Shanghai, China). They were grown in DMEM enriched with 10\% FBS, $100 \mathrm{U} / \mathrm{mL}$ penicillin and $100 \mathrm{mg} / \mathrm{mL}$ streptomycin. We grew the cells in a humidified environment, at $37^{\circ} \mathrm{C}, 5 \% \mathrm{CO}_{2}$, in an incubator.

\section{Transfection}

Lentiviral IARS2 shRNAs and the negative control were designed and produced by Hanbio (Shanghai, China). The target sequences of anti-IARS2 shRNAs were: shRNA-1, 5 '-cagtggtttataaacatcacggata-3' and shRNA-2, 5'-cagcaaggaagagagcaccttataa-3'. They were constructed using pHBLV-U6-ZsGreen-Puro vector. Cells were cultured in $6 \mathrm{~cm}$ dishes and transfected with $10 \mu \mathrm{L}$ of the lentivirus in the presence of polybrene at a final concentration of 10 $\mu \mathrm{g} / \mathrm{mL}$ for 48 hours. Stably transfected cells were selected using $3 \mu \mathrm{g} / \mathrm{mL}$ puromycin for 7 days. RT-qPCR and western blot analyses were used to confirm knockdown.

\section{Western blot analysis}

Total proteins were isolated from HUVEC and the BCA protein assay Kit (Beyotime, Cat No. P0012) was employed to quantify them. Thirty micrograms of each protein sample were resolved by $10 \%$ SDS-PAGE. We transfer-embedded the proteins onto PVDF membranes. After blocking with 5\% milk for two hours, we incubated the membranes overnight with the indicated primary antibodies at the membranes at $4^{\circ} \mathrm{C}$. After that, we conjugated the membranes with the respective secondary antibodies at RT for two hours. Signal was then developed using FluoChem E system (Bio-Techne) and quantified using ImageJ. The following antibodies were used at 1:1000: rabbit anti-IARS2 (Proteintech, Cat No. 17170-1-AP), rabbit anti- $\beta$-catenin (Santa Cruz Biotechnology, Cat No. sc-7963), rabbit anti-AKT (Proteintech, Cat No. 10176-2-AP), rabbit anti-Phospho-Akt (Cell Signaling Technology, Cat No. 4060S), rabbit anti-GSK3 $\beta$ (Proteintech, Cat No. 22104-1-AP), mouse anti- $\beta$-actin (Cell Signaling Technology, Cat No. 4967S), rabbit anti-VEGF (Bioss Biotech, Cat No. bs-1665R), rabbit anti-extracellular signal-regulated kinase (ERK1/2) (Cell Signaling Technology, Cat No. 9102S), and rabbit anti-phospho-ERK1/2 (Cell Signaling Technology, Cat No. 4376S).

\section{Cell proliferation and colony formation} To test the proliferative impacts of IARS2 knockdown, we planted $100 \mu \mathrm{L}$ of $3 \times 10^{3}$ cells/well onto in 96 -well culture plates and grew them for five days. Cell proliferation was then measured by adding $10 \mu \mathrm{L}$ CCK-8 reagent (Dojindo Laboratories, Cat No. CK04) into every well and incubating the plate for two hours at $37^{\circ} \mathrm{C}$, followed by $450 \mathrm{~nm}$ absorbance reading on a microplate reader. Colony formation analysis was done by culturing 400 cells/well in $6 \mathrm{~cm}$ dishes for two weeks, and successive staining was accomplished using crystal violet. We counted the colonies with $>50$ cells.

\section{Cell migration assessment}

We employed the Wound healing test to inspect cell migration. We planted $4 \times 10^{5}$ cells/well in 6 -well plates and incubated overnight to $90 \%$ confluence. We then a made a wound via scratching the monolayer using a $200 \mu \mathrm{L}$ pipette tip. Cell migration over the wounded area was then measured after 24 hours.

Transwell chambers without Matrigel were used to measure cell migration capacity. The amount of $5 \times 10^{4} /$ well in $200 \mu \mathrm{L}$ of serum-free medium was cultured on the upper chamber for 48 hours. Subsequently, membrane fixation was accomplished using $100 \%$ methanol for 10 minutes. Successive staining was carried out using $0.1 \%$ crystal violet for 30 minutes and cell migration was examined under an inverted microscope.

\section{Tube formation assessment}

The amount of $50 \mu \mathrm{L} /$ well of Matrigel was used to coat 96 -well plates. After that, we incubated the plates at $37^{\circ} \mathrm{C}$ for $30 \mathrm{~min}-$ utes. We subsequently added HUVECs $\left(4 \times 10^{4} \mathrm{cells} /\right.$ well $)$ in $100 \mu \mathrm{L}$ serum-free DMEM on the solidified Matrigel. After 24 hour incubation, the capillary-like structures were then imaged under a digital microscope system. 


\section{Real time quantitative polymerase chain reaction analysis}

RNA purification from the cells was accomplished using TRIzol reagent following the protocol provided by the manufacturer. Reverse transcription was done using PrimeScript ${ }^{\mathrm{TM}} \mathrm{RT}$ Master Mix (Takara, Cat No RR036B). RT-qPCR analysis was carried out on an ABI 7500 real-time PCR machine (Applied Biosystems) using SYBR Green Premix Ex Taq ${ }^{\mathrm{Tm}}$ (Takara, Cat No RR820B). The amplification conditions were $95^{\circ} \mathrm{C}$ for 30 seconds, 40 cycles at $95^{\circ} \mathrm{C}$ for 5 seconds, and $60^{\circ} \mathrm{C}$ for 34 seconds. The primer sequences for IARS 2 and $\beta$-actin were: IARS2, forward primer, 5'-actgcccgaagtttgtggg-3', reverse primer, 5' -cggtatctgccactattcgagtt- $3^{\prime}$; and $\beta$-actin, forward primer, $5^{\prime}$-tgtaaggttgtccagttcaaaagact-3', reverse primer, 5'-ccagctcaccatggatgatg-3'. $\beta$-actin was employed as a normalization gene and data were assessed using the delta-delta $\mathrm{Ct}$ approach.

\section{Statistical analysis}

GrahpPad Prism 7.0 software was employed to conduct the statistical analyses. All data were expressed as mean $\pm \mathrm{SD}$ of three independent experiments. We employed the two-tailed student's t-test to compare the differences between groups. $\mathrm{p}<05$ signified statistical significance.

\section{RESULTS}

\section{Expression of Isoleucyl-TRNA Synthetase 2 was repressed by short hairpin RNAs-IARS2 in human umbilical vein endothelial cells}

To assess the role of IARS2, we knocked down IARS2 in HUVEC cells using two specific shRNAs (shRNA1 and 2) and examined IARS2 titers by western blot analysis, as well as RT-qPCR assays (Figure 1). Because shRNA1-IARS2 had greater knockdown efficiency, it was used in the entire downstream analysis.

\section{Isoleucyl-TRNA Synthetase 2 knockdown suppresses human umbilical vein endothelial cells proliferation, migration, and angiogenesis}

CCK-8 analysis revealed that IARS2-silenced HUVECs grew slower than control cells (Figure 2A). Colony formation assays showed that IARS2-silenced cells formed significantly fewer colonies compared to control cells (Figure 2B-C). Similar observations were made from wound healing assays (Figure 2D-E). Additionally, Transwell ${ }^{\circledR}$ migration assay (without Matrigel) revealed suppressed cell migration upon shRNA1-IARS2 knockdown compared to the control group after 48 hours (Figure 2F-G).
Tube-formation assay constitutes a well-documented in vitro test for angiogenesis and is centered on the capacity of HUVECs to develop three-dimensional capillary-like tubular structures. To evaluate the role of IARS2 in angiogenesis, control or IARS1-silenced HUVECs were planted on a thin layer of Matrigel and fixed after 24 hours. Tube formation analysis showed that IARS2 knockdown significantly suppressed tube formation, the branches of capillary tube in HUVECs knockdown IARS2 were significantly reduced by $67.0 \%$ compared to the control group (Figure 2H), suggesting that IARS2 knockdown suppressed angiogenesis.

\section{Isoleucyl-TRNA Synthetase 2 knockdown alters cell signaling pathways}

To elucidate the mechanism by which IARS2 knockdown suppressed HUVECs proliferation, migration, and angiogenesis, we examined cell signaling pathways associated with these processes using a western blot analysis. This analysis found that AKT, ERK and GSK3- $\beta / \beta$-catenin signaling pathways may be involved in IARS2-mediated functions. IARS2 knockdown suppressed phosphorylation of ERK1/2 and AKT. IARS2 knockdown also decreased the expression of VEGF, GSK-3 $\beta$, and $\beta$-catenin (Figure 3).

\section{DISCUSSION}

Multiple studies have shown that tumors are angiogenesis dependent and angiogenesis is a well-known mediator of tumor growth and metastasis. Thus, anti-angiogenic therapies are promising anti-cancer strategies ${ }^{10}$.

ARSs expression profiles have been reported as potential cancer prognosis biomarkers because they are associated with overall patient survival for all types of cancer on the human protein atlas repository ${ }^{11}$. Multiple studies have implicated the role of ARSs in tumorigenesis, angiogenesis, and inflammation ${ }^{12}$, highlighting their potential as anti-cancer targets.

Past studies suggest that ARSs, including tyrosyl-tRNA synthetase (YARS), tryptophanyl-tRNA synthetase (WARS), threonyl-tRNA synthetase (TARS), seryl-tRNA synthetase (SARS), and glutamyl-prolyl-tRNA synthetase (EPRS) influence angiogenesis. SARS and EPRS are thought to influence angiogenesis by modulating the expression of angiogenic factors, including VEGF ${ }^{13}$. However, few studies have examined the role of IARS2 in angiogenesis.

IARS2 is involved in various diseases, i.e., liver disease, hypotonia, and intellectual disability ${ }^{14}$. IARS2 silencing suppresses cell proliferation in non-small cell lung cancer, induces cell cycle interruption, and enhances apoptosis ${ }^{15}$. In this study, we found that IARS2 knockdown suppresses HUVEC 


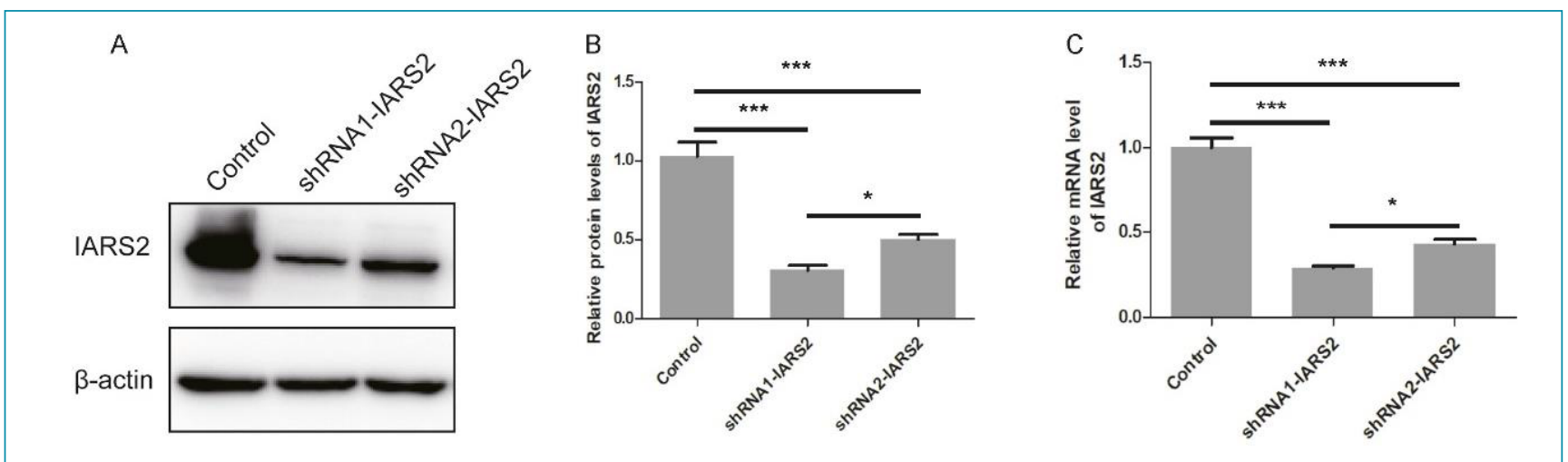

Figure 1. Expression of Isoleucyl-TRNA Synthetase 2 was repressed by short hairpin RNAs 2 in human umbilical vein endothelial cells. (A) Human umbilical vein endothelial cells were infected with 2 anti- Isoleucyl-TRNA Synthetase 2 lentiviral short hairpin RNAs or control, and Isoleucyl-TRNA Synthetase 2 protein level measured by western blotting. (B) Measurement of IsoleucylTRNA Synthetase 2 silencing efficiency by protein level analysis in the human umbilical vein endothelial cells. (C) Real time quantitative polymerase chain confirmed Isoleucyl-TRNA Synthetase 2 knockdown. Data are indicated as the mean $\pm S D$ of three independent assays $\left({ }^{*} p<0.05,{ }^{*} p<0.01\right.$, and $\left.{ }^{* * *} p<0.001\right)$.

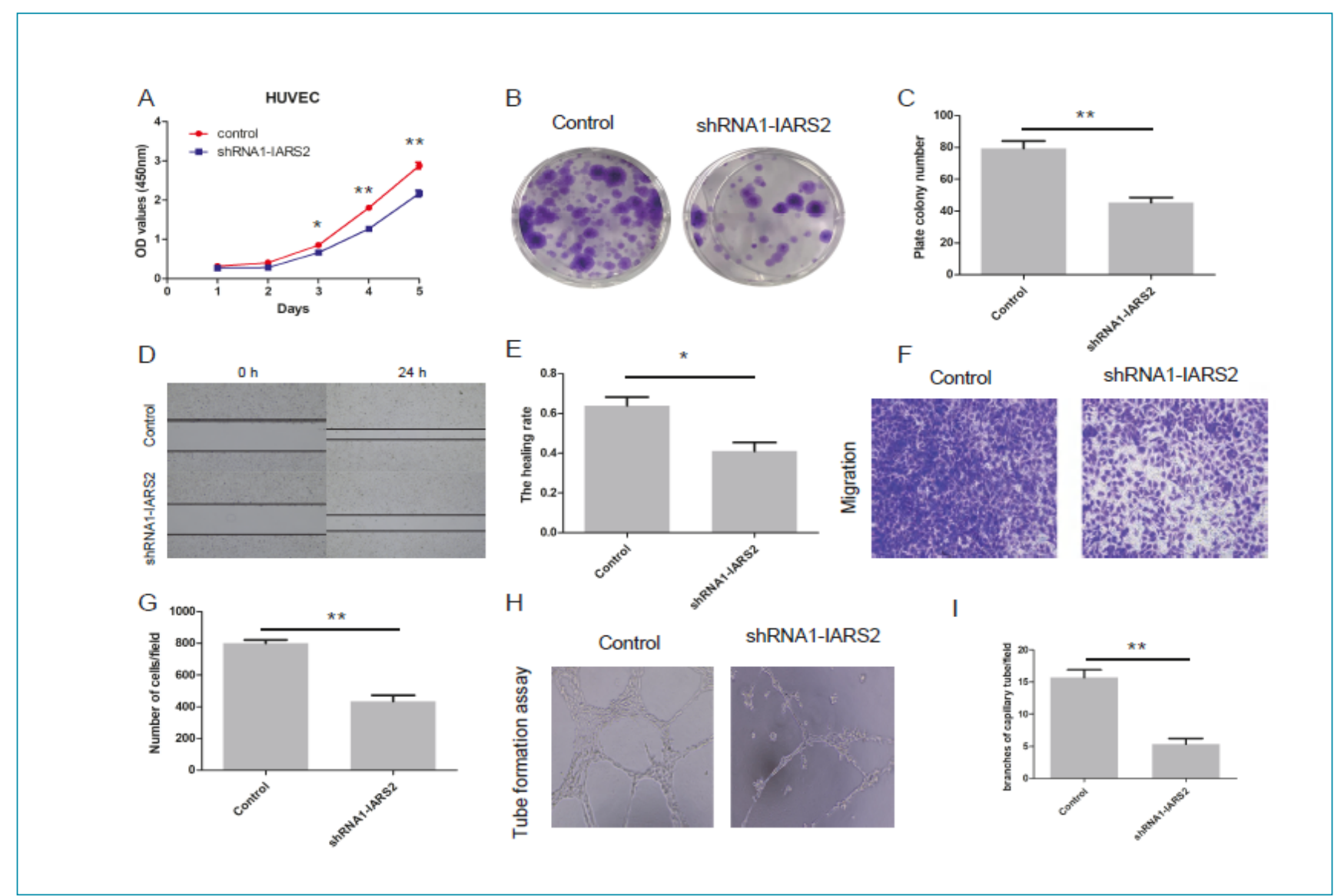

Figure 2. Isoleucyl-TRNA Synthetase 2 knockdown silencing repressed proliferation, migration and angiogenesis of human umbilical vein endothelial cells. (A) Cell proliferation was measured using CCK8 analysis. (B) Cell colony formation assay images. (C) Quantitative analyses of the relative colony number. (D) Human umbilical vein endothelial cells migration assay at 0 and $24 \mathrm{~h}$ post-wounding. (E) Quantification of the above-mentioned assay. (F) Human umbilical vein endothelial cells migration upon incubation in permeable supports for $48 \mathrm{~h}$ was assessed using crystal violet staining. (G) Quantification of the aforementioned assay. (H) Illustrative images of tube formation in Human umbilical vein endothelial cells treated with control or Isoleucyl-TRNA Synthetase 2 short hairpin RNAs. (I) The branches of capillary tube formed by Human umbilical vein endothelial cells. Data are indicated as mean \pm SD of three independent experiments $\left({ }^{*} p<0.05,{ }^{*} p<0.01\right.$, and $\left.{ }^{* * *} p<0.001\right)$. 
A

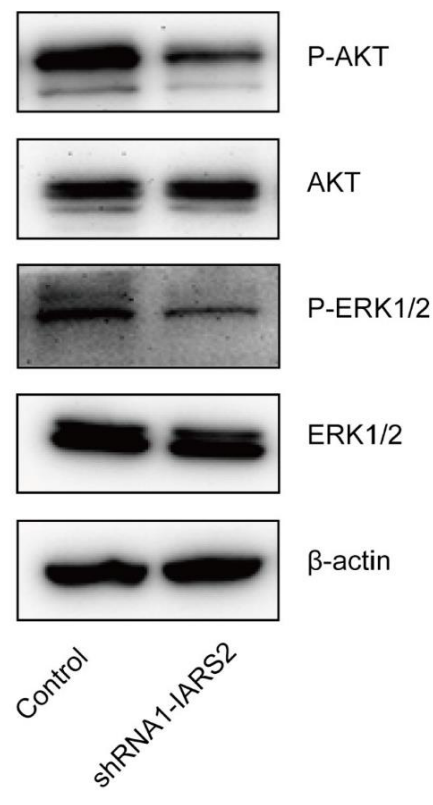

B
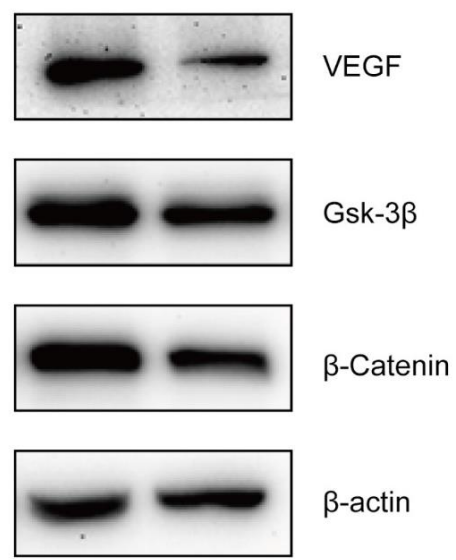

$\beta$-actin

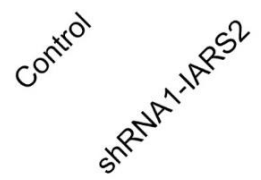

Figure 3. Alterations in signaling pathways upon Isoleucyl-TRNA Synthetase 2 knockdown. (A) Western blot analyses of the phosphorylation of extracellular signal-regulated kinase $1 / 2$ and the phosphorylation of protein-serine- threonine kinase in Isoleucyl-TRNA Synthetase 2 knockdown human umbilical vein endothelial cells. (B) Western blot analyses of vascular endothelial growth factor, GSK-3 $\beta$ and $\beta$-catenin, upon Isoleucyl-TRNA Synthetase 2 knockdown versus control knockdown. Data are shown as mean \pm SD of three independent experiments $\left({ }^{*} p<0.05,{ }^{*} p<0.01\right.$, and $\left.{ }^{* * *} p<0.001\right)$.

proliferation, migration, and angiogenesis. IARS2 knockdown also suppressed phosphorylation of ERK1/2, as well as AKT, and reduced expression of VEGF, GSK-3 $\beta$, and $\beta$-catenin. These results enhance our understanding of angiogenic mechanisms and highlight IARS2 as a potential anti-angiogenic target.

Multiple studies have implicated Wnt/ $\beta$-catenin, PI3K/ AKT, Ras/Raf/MEK/ERK, as well as VEGF, in tumor cell metabolism, growth, proliferation, survival, as well as angiogenesis ${ }^{16}$. AKT and ERK pathway inhibitors suppress angiogenesis ${ }^{17}$. Our data show IARS2 knockdown suppresses phosphorylation of ERK1/2 and AKT. VEGF is a crucial angiogenic factor with central roles in physiologic and pathologic tumor angiogenesis. It modulates all the main steps of angiogenesis-linked processes consisting of endothelial cell proliferation, migration, and tube formation ${ }^{18}$. Here, we find that VEGF protein levels are remarkably reduced by IARS2 knockdown. Wnt/ $\beta$-catenin signaling also mediates angiogenesis, vascular remodeling, and differentiation and Wnt signaling inhibition may suppress angiogenesis. GSK3 $\beta$ directly phosphorylates $\beta$-catenin at multiple sites causing its degradation and thus negatively modulating Wnt $/ \beta$-catenin signaling ${ }^{19}$. IARS2 knockdown inhibited $\beta$-catenin and GSK3 $\beta$ protein levels. However, GSK3 $\beta$ may negatively correlate with $\beta$-catenin. Thus, the crosstalk between signaling pathways is complex and evidences the need for additional studies.

\section{CONCLUSIONS}

In summary, we have shown, for the first time, the critical role of IARS2 in HUVECs. Our data show that IARS2 knockdown suppresses HUVEC proliferation, migration, and angiogenesis. We have also shown that IARS2 knockdown suppresses phosphorylation of ERK1/2 and AKT, as well as expression of VEGF, GSK-3 $\beta$, and $\beta$-catenin. Together, these data highlight IARS2 as a potential antitumor anti-angiogenic target.

\section{AUTHORS" CONTRIBUTIONS}

YMY: Conceptualization. LX: Conceptualization, Formal Analysis. HRL: Writing - original draft. TQZ: Writing review \& editing. GQ: Supervision. LFL: Supervision. MHW: Funding acquisition. 


\section{REFERENCES}

1. Folkman J. Role of angiogenesis in tumor growth and metastasis. Semin Oncol. 2002;29(6 Suppl 16):15-8. https:// doi.org/10.1016/S0093-7754(02)70065-1

2. Weis $\mathrm{SM}$, Cheresh DA. Tumor angiogenesis: molecular pathways and therapeutic targets. Nat Med. 2011;17(11):1359-70. https://doi.org/10.1038/nm.2537

3. Shahneh FZ, Baradaran B, Zamani F, Aghebati-Maleki L. Tumor angiogenesis and anti-angiogenic therapies. Hum Antibodies. 2013;22(1-2):15-9. https://doi.org/10.3233/HAB-130267

4. Jośko J, Gwóźdź B, Jedrzejowska-Szypułka H, Hendryk S. Vascular endothelial growth factor (VEGF) and its effect on angiogenesis. Med Sci Monit. 2000;6(5):1047-52. PMID: 11208453

5. Mander KA, Finnie JW. Tumour angiogenesis, anti-angiogenic therapy and chemotherapeutic resistance. Aust Vet J. 2018;96(10):371-8. https://doi.org/10.1111/avj.12747

6. Kwon NH, Fox PL, Kim S. Aminoacyl-tRNA synthetases as therapeutic targets. Nat Rev Drug Discov. 2019;18(8):629-50. https://doi.org/10.1038/s41573-019-0026-3

7. Kim S, You S, Hwang D. Aminoacyl-tRNA synthetases and tumorigenesis: more than housekeeping. Nat Rev Cancer. 2011;11(10):708-18. https://doi.org/10.1038/nrc3124

8. Zhong $L$, Zhang $Y$, Yang $J Y$, Xiong $L F$, Shen $T$, Sa $Y L$, et al. Expression of IARS2 gene in colon cancer and effect of its knockdown on biological behavior of RKO cells. Int J Clin Exp Pathol. 2015;8(10):12151-9. PMID: 26722399

9. Fang Z, Wang $X$, Yan Q, Zhang S, Li Y. Knockdown of IARS2 suppressed growth of gastric cancer cells by regulating the phosphorylation of cell cycle-related proteins. Mol Cell Biochem. 2018;443(1-2):93-100. https://doi.org/10.1007/ s11010-017-3213-8

10. Teleanu RI, Chircov C, Grumezescu AM, Teleanu DM. Tumor Angiogenesis and Anti-Angiogenic Strategies for Cancer Treatment. J Clin Med. 2019;9(1):84. https://doi.org/10.3390/ jcm9010084
11. Uhlen M, Zhang C, Lee S, Sjöstedt E, Fagerberg L, Bidkhori G, et al. A pathology atlas of the human cancer transcriptome. Science. 2017;357(6352):eaan2507. https://doi.org/10.1126/ science.aan2507

12. Kim D, Kwon NH, Kim S. Association of aminoacyl-tRNA synthetases with cancer. Top Curr Chem. 2014;344:207-45. https://doi.org/10.1007/128_2013_455

13. Mirando A, Francklyn C, Lounsbury K. Regulation of angiogenesis by aminoacyl-tRNA synthetases. Int J Mol Sci. 2014;15(12):23725-48. https://doi.org/10.3390/ijms151223725

14. Smigiel R, Biela M, Biernacka A, Stembalska A, Sasiadek M, Kosinska J, et al. New evidence for association of recessive IARS gene mutations with hepatopathy, hypotonia, intellectual disability and growth retardation. Clin Genet. 2017;92(6):671-3. https://doi.org/10.1111/cge.13080

15. Yin J, Liu W, Li R, Liu J, Zhang Y, Tang W, et al. IARS2 silencing induces non-small cell lung cancer cells proliferation inhibition, cell cycle arrest and promotes cell apoptosis. Neoplasma. 2016;63(1):64-71. https://doi.org/10.4149/neo_2016_008

16. Chiu H, Li C, Yiang G, Tsai A, Wu M. Epithelial to mesenchymal transition and cell biology of molecular regulation in endometrial carcinogenesis. J Clin Med. 2019;8(4):439. https://doi. org/10.3390/jcm8040439

17. Zhao L, Bode AM, Cao Y, Dong Z. Regulatory mechanisms and clinical perspectives of miRNA in tumor radiosensitivity. Carcinogenesis. 2012;33(11):2220-7. https://doi.org/10.1093/ carcin/bgs235

18. Melincovici CS, Boșca AB, Șușman S, Mărginean M, Mihu $C$, Istrate $M$, et al. Vascular endothelial growth factor (VEGF) - key factor in normal and pathological angiogenesis. Rom J Morphol Embryol. 2018;59(2):455-67. PMID: 30173249

19. Vallée $A$, Guillevin $R$, Vallée J. Vasculogenesis and angiogenesis initiation under normoxic conditions through Wnt $\beta$-catenin pathway in gliomas. Rev Neurosci. 2018;29(1):71-91. https:// doi.org/10.1515/revneuro-2017-003 